\title{
Influence of the confinement potential on the electron-hole-pair states in semiconductor microcrystallites
}

\author{
D. B. Tran Thoai* \\ Fritz-Haber-Institut der Max-Planck-Gesellschaft zur Föderung der Wissenschaften, \\ Faradayweg 4-6, D-1000 Berlin 33, Federal Republic of Germany \\ Y. Z. Hu \\ Department of Physics, University of Arizona, Tucson, Arizona 85721 \\ S. W. Koch \\ Optical Sciences Center and Department of Physics, University of Arizona, Tucson, Arizona 85721
}

(Received 5 February 1990; revised manuscript received 9 July 1990)

\begin{abstract}
The energies of the two energetically lowest dipole-allowed electron-hole-pair states in semiconductor microcrystallites are computed variationally. Details of the quantum confinement conditions, such as the finite value of the quantum confinement potential and the different effective electron-hole masses inside and outside the crystallites, are considered explicitly. Significant deviations from the infinite-potential approximation are obtained.
\end{abstract}

\section{INTRODUCTION}

Three-dimensional quantum confinement effects in semiconductor microcrystallites occur when the particle size becomes comparable to the exciton Bohr radius. The confinement gives rise to interesting new effects which have attracted considerable attention recently. ${ }^{1-14}$ The research has concentrated mainly on optically excited compound semiconductor clusters in glasses or other dielectrics, ${ }^{1,3,4,8-10,14}$ and on colloidal semiconductors precipitated in liquids. ${ }^{2,4,6,7}$ as well as on quantum dots in organic films or inside zeolites. ${ }^{13}$ The first theoretical descriptions of three-dimensional excitonic quantum confinement were given by Efros and Efros ${ }^{1}$ and by Brus. ${ }^{2}$ These authors discussed the basic optical properties of semiconductor microcrystallites as functions of crystallite size. Brus ${ }^{2}$ calculated the energy of the lowest electronhole-pair state, approximating the exciton wave function by a few configurations of the electron-hole $s$ wave functions confined in a sphere. Recent variational calculations achieved some improvements in determining the energy of the lowest electron-hole-pair state. ${ }^{5,6}$ However, for crystallite radii comparable to the bulk exciton Bohr radius, theoretical results ${ }^{5,6}$ overestimate the confinement-induced blue shift in comparison with experimental results. ${ }^{3,4}$ We believe that one reason for this overestimation is the assumption of an infinite-potential barrier in the hard-wall model, which yields a too large kinetic energy of the electron and hole, particularly in the case of small crystallite radii. In this paper, we compute the energy of the two lowest dipole allowed quantumconfined electron-hole-pair states in semiconductor microcrystallites taking into account a finite barrier for the confining potential. To determine the wave function outside the quantum dots we need to model the environment of the microcrystallites. For the case of quantum dots in a liquid, we assume that the electrons are mobile outside the quantum dot and use the free-electron mass $m_{0}$ for the electrons in the liquid. For the case of quantum dots in glass, we model the electron states in the glass as localized states with very low mobility and correspondingly large electron mass. We present a systematic study of the parameter dependence of the computed quantumconfined pair states.

\section{VARIATIONAL CALCULATIONS}

In the framework of the effective-mass approximation, the electron-hole-pair Hamiltonian is given by

$$
\begin{aligned}
H= & -\frac{\hbar^{2} \nabla_{e}^{2}}{2 m_{e}}-\frac{\hbar^{2} \nabla_{h}^{2}}{2 m_{h}}-\frac{e^{2}}{\epsilon\left|r_{e}-r_{h}\right|} \\
& +V_{e}\left(r_{e}\right)+V_{h}\left(r_{h}\right)+E_{g},
\end{aligned}
$$

with the confinement potential

$$
V_{i}\left(r_{i}\right)=\left\{\begin{array}{l}
0 \text { for } r_{i} \leq R \quad(i=e, h) \\
V_{0 i} \text { for } r_{i} \geq R \quad(i=e, h) .
\end{array}\right.
$$

Here $R$ is the crystallite radius; $\epsilon$ is the background dielectric constant of the semiconductor material; $E_{g}$ is the band-gap energy of the bulk semiconductor material, $m_{e}, r_{e}$, and $V_{0 e}$ and $m_{h}, r_{h}$, and $V_{0 h}$ are the effective mass, position, and potential-well height of the electron and the hole, respectively. The contribution of the surface polarization terms is small, ${ }^{7}$ and we neglect it here for the sake of simplicity.

\section{A. Ground state}

In order to calculate the ground-state energy of the electron-hole pair we choose the trial wave function as 


$$
\psi\left(\mathbf{r}_{e}, \mathbf{r}_{h}\right)=e^{-\lambda_{s}\left|\mathbf{r}_{e}-\mathbf{r}_{h}\right|} \phi_{e, s}\left(r_{e}\right) \phi_{h, s}\left(r_{h}\right),
$$

where $\lambda_{S}$ is the variational parameter. The functions $\phi_{e}$ $\left(\phi_{h}\right)$ for the electron (hole) are the radial eigenfunctions corresponding to the lowest energy state in a spherical well of finite depth, i.e.,

$$
\phi_{i, s}\left(r_{i}\right)=\left\{\begin{array}{c}
\frac{\sin \left(k_{i, s} r_{i}\right)}{k_{i, s} r_{i}} \quad \text { for } r_{i} \leq R \quad(i=e, h) \\
\frac{\sin \left(k_{i, s} R\right)}{k_{i, s} r_{i}} e^{-q_{i, s}\left(r_{i}-R\right)} \\
\text { for } r_{i} \geq R(i=e, h)
\end{array}\right.
$$

where we defined

$$
\begin{aligned}
k_{i, s}^{2} & =\frac{2 m_{i, 1} E_{i, s}}{\hbar^{2}}, \\
q_{i, s}^{2} & =\frac{2 m_{i, 2} V_{0, i}}{\hbar^{2}}-k_{i, s}^{2} \frac{m_{i, 2}}{m_{i, 1}},
\end{aligned}
$$

and $m_{e, 1}, m_{h, 1}$, and $m_{e, 2}, m_{h, 2}$, denote the effective mass of the electron and hole inside and outside the microcrystallite. The effective masses inside and outside the quantum dots may be very different, describing semiconductor band electrons with an effective mass inside, and quasifree electrons in a liquid ( $m_{e, 2} \cong m_{0}$ ) or electrons in more or less localized states in a glass matrix, $m_{e, 2} \gg m_{0}$, outside, respectively. The variational wave function (3) was chosen because it approaches the hydrogenic $1 s$ wave function for the relative electron-hole motion for $R \rightarrow \infty$ and $\lambda_{s} \rightarrow 1 / a_{B}$, where $a_{B}$ is the bulk-exciton Bohr radius.

From the boundary conditions that $\phi_{i}\left(r_{i}\right)$ and $\left(1 / m_{i}\right) \partial \phi_{i}\left(r_{i}\right) / \partial r_{i}$ are both continuous at $r_{i}=R$, we obtain

$\tan \left(k_{i, s} R\right)=\frac{k_{i, s} R}{1-\left(1+q_{i, s} R\right) m_{i, 1} / m_{i, 2}} \quad(i=e, h)$,

which determines the ground-state energy $E_{e, s}\left(E_{h, s}\right)$ of the noninteracting electron (hole) in the potential well.

The ground-state energy of the interacting electronhole pair is computed from the expectation value of the Hamiltonian (1) with the trial wave function (3). After a straightforward but lengthy calculation, we obtain

$$
\begin{aligned}
E_{s}= & \frac{\hbar^{2}}{2 m_{e, 1}}\left(\lambda_{s}^{2}+k_{e, s}^{2}\right)+\frac{\hbar^{2}}{2 m_{h, 1}}\left(\lambda_{s}^{2}+k_{h, s}^{2}\right)-\frac{e^{2} C_{s}}{\epsilon N_{s}}+\frac{\hbar^{2} \lambda_{s}^{2}}{4 N_{s}}\left[\left(\frac{1}{m_{e, 1}}-\frac{1}{m_{e, 2}}\right) A_{e, s}+\left(\frac{1}{m_{h, 1}}-\frac{1}{m_{h, 2}}\right) A_{h, s}\right] \\
& -\frac{\hbar^{2} \lambda_{s}^{2}}{2 N_{s}}\left[\left(\frac{1}{m_{e, 1}}-\frac{1}{m_{e, 2}}\right) B_{e, s}+\left(\frac{1}{m_{h, 1}}-\frac{1}{m_{h, 2}}\right) B_{h, s}\right],
\end{aligned}
$$

where we introduced the following quantities:

$$
\begin{aligned}
& N_{s}=\int_{0}^{\infty} d r_{e} \int_{0}^{\infty} d r_{h} r_{e} r_{h} \phi_{e, s}^{2}\left(r_{e}\right) \phi_{h, s}^{2}\left(r_{e}\right) F_{1}\left(2 \lambda_{s}, r_{e}, r_{h}\right) \\
& C_{s}=\int_{0}^{\infty} d r_{e} \int_{0}^{\infty} d r_{h} r_{e} r_{h} \phi_{e, s}^{2}\left(r_{e}\right) \phi_{h, s}^{2}\left(r_{h}\right) \\
& \quad \times\left(e^{-2 \lambda_{s}\left|r_{e}-r_{h}\right|}-e^{-2 \lambda_{s}\left|r_{e}+r_{h}\right|}\right) \\
& A_{e, s}=\phi_{e, s}^{2}(R) \int_{0}^{\infty} d r_{h} r_{h} \phi_{h, s}^{2}\left(r_{h}\right) S_{1}\left(2 \lambda_{s}, R, r_{h}\right) \\
& B_{e, s}=\int_{R}^{\infty} d r_{e} r_{e} \int_{0}^{\infty} d r_{h} r_{h} \phi_{e, s}^{2}\left(r_{e}\right) \phi_{h, s}^{2}\left(r_{h}\right) F_{1}\left(2 \lambda_{s}, r_{e}, r_{h}\right)
\end{aligned}
$$

and, correspondingly, $A_{h, s}=\left.A_{e, s}\right|_{e \leftrightarrow h}, \quad B_{h, s}=\left.B_{e, s}\right|_{e \leftrightarrow h}$. Furthermore, we defined

$$
S_{1}\left(\lambda, x_{1}, x_{2}\right)=\frac{\partial}{\partial x_{1}} \frac{F_{1}\left(\lambda, x_{1}, x_{2}\right)}{x_{1}}
$$

with

$$
\begin{aligned}
& \frac{F_{1}\left(\lambda, x_{1}, x_{2}\right)}{2 x_{1} x_{2}} \\
& \quad=\int_{-1}^{1} d y y^{i-1} \exp \left[-\lambda\left(x_{1}^{2}+x_{2}^{2}-2 x_{1} x_{2} y\right)^{1 / 2}\right],
\end{aligned}
$$

and

$$
\begin{aligned}
& \frac{D_{1}\left(\lambda, x_{1}, x_{2}\right)}{2 x_{1} x_{2}} \\
& =\int_{-1}^{1} d y y^{i-1} \exp \left[-\lambda\left(x_{1}^{2}+x_{2}^{2}-2 x_{1} x_{2} y\right)^{1 / 2}\right] \\
& \quad \times\left(x_{1}^{2}+x_{2}^{2}-2 x_{1} x_{2} y\right)^{1 / 2}
\end{aligned}
$$

$\frac{G_{i}\left(\lambda, x_{1}, x_{2}\right)}{2 x_{1} x_{2}}$

$$
=\int_{-1}^{1} d y \frac{y^{i-1} \exp \left[-\lambda\left(x_{1}^{2}+x_{2}^{2}-2 x_{1} x_{2} y\right)^{1 / 2}\right]}{\left(x_{1}^{2}+x_{2}^{2}-2 x_{1} x_{2} y\right)^{1 / 2}}
$$


As usual, we now have to determine the variational parameter $\lambda_{s}$ by minimizing $E_{s}$.

\section{B. First excited state}

For the first excited state we need a variational wave function that (i) can reduce to the strong-confinement product of $p$-state functions for the electron and hole for $R \rightarrow 0$, and (ii) can reproduce the $2 s$ wave function for the electron-hole relative motion for large quantum dots. We choose

$$
\begin{aligned}
\Phi\left(\mathbf{r}_{e}, \mathbf{r}_{h}\right)= & \phi_{e, p}\left(r_{e}\right) \phi_{h, p}\left(r_{h}\right) \cos (\gamma) \\
& \times\left(\delta-\lambda_{p}\left|\mathbf{r}_{e}-\mathbf{r}_{h}\right|\right) e^{-\lambda_{p}\left|\mathbf{r}_{e}-\mathbf{r}_{h}\right|},
\end{aligned}
$$

where

$$
\phi_{i, p}\left(r_{i}\right)=\left\{\begin{array}{c}
\frac{\sin \left(k_{i, p} r_{i}\right)}{k_{i, p}^{2} r_{1}^{2}}-\frac{\cos \left(k_{i, p} r_{i}\right)}{k_{i, p} r_{i}} \\
\text { for } r_{i} \leq R \quad(i=e, h) \\
{\left[\sin \left(k_{i, p} R\right)-k_{i, p} R \cos \left(k_{i, p} R\right)\right] q_{i, p}^{2}} \\
\left(q_{i, p} R+1\right) k_{i, p}^{2} \\
\times \frac{e^{-q_{i, p}\left(r_{1}-R\right)}}{q_{i, p} r_{i}}\left(\frac{1}{q_{i, p} r_{i}}+1\right) \\
\text { for } r_{i} \geq R \quad(i=e, h) .
\end{array}\right.
$$

With $\gamma$ in Eq. (17) we denote the angle between $\mathbf{r}_{e}$ and $\mathbf{r}_{h}$, and $\delta$ and $\lambda_{p}$ are the variational parameters. One of these parameters is fixed by the condition that the wave function (17) has to be orthogonal to the ground-state wave function (3). We obtain

$$
\delta=\lambda_{p} \frac{\int_{0}^{\infty} d r_{e} \int_{0}^{\infty} d r_{h} r_{e} r_{h} \phi_{e, s}\left(r_{e}\right) \phi_{e, p}\left(r_{e}\right) \phi_{h, s}\left(r_{h}\right) \phi_{h, p}\left(r_{h}\right) F_{2}\left(\lambda_{s}+\lambda_{p}, r_{e}, r_{h}\right)}{\int_{0}^{\infty} d r_{e} \int_{0}^{\infty} d r_{h} r_{e} r_{h} \phi_{e, s}\left(r_{e}\right) \phi_{e, p}\left(r_{e}\right) \phi_{h, s}\left(r_{h}\right) \phi_{h, p}\left(r_{h}\right) D_{2}\left(\lambda_{s}+\lambda_{p}, r_{e}, r_{h}\right)} .
$$

As for the ground-state calculation, we use the boundary conditions for the wave function (17) to determine the excited-state energies of the noninteracting electrons and holes from the relation

$$
\tan \left(k_{i, p} R\right)=\frac{k_{i, p} R}{1+\frac{\left(k_{i, p} R\right)^{2}}{\frac{m_{i, 1}}{m_{i, 2}} \frac{2+2 q_{i, p} R+q_{i, p}^{2} R^{2}}{1+q_{i, p} R}-2}}
$$

where $k_{i, p}$ and $q_{i, p}$ are defined as in Eqs. (5) and (6) with the index $s$ being replaced by $p$. The expectation value of the Hamiltonian (1) with the wave function (17) gives the excited-state energy of the interacting electron-hole pair as

$$
\begin{aligned}
E_{p}= & \frac{\hbar^{2}}{2 m_{e, 1}}\left[\lambda_{e, p}^{2} \frac{D_{e, p}}{N_{p}}+k_{e, p}^{2}\right]+\frac{\hbar^{2}}{2 m_{h, 1}}\left[\lambda_{h, p}^{2} \frac{D_{h, p}}{N_{p}}+k_{h, p}^{2}\right]-\frac{e^{2} C_{p}}{\epsilon N_{p}} \\
& +\frac{\hbar^{2} \lambda^{2}}{4 ! N_{p}}\left[\left(\frac{1}{m_{e, 1}}-\frac{1}{m_{e, 2}}\right) A_{e, p}+\left(\frac{1}{m_{h, 1}}-\frac{1}{m_{h, 2}}\right) A_{h, p}\right]-\frac{\hbar^{2} \lambda^{2}}{2 N_{p}}\left[\left(\frac{1}{m_{e, 1}}-\frac{1}{m_{e, 2}}\right) B_{e, p}+\left[\frac{1}{m_{h, 1}}-\frac{1}{m_{h, 2}}\right] B_{h, p}\right],
\end{aligned}
$$

where

$$
\begin{aligned}
D_{e, p}=\int_{0}^{\infty} d r_{e} \int_{0}^{\infty} d r_{h} r_{e} r_{h} \phi_{e, p}^{2}\left(r_{e}\right) \phi_{h, p}^{2}\left(r_{h}\right)\{ & {\left[(1+\delta)^{2}+\lambda_{p}^{2}\left(r_{e}^{2}+r_{h}^{2}\right)\right] F_{3}\left(2 \lambda_{p}, r_{e}, r_{h}\right) } \\
& \left.-2 \lambda_{p}^{2} r_{e} r_{h} F_{4}\left(2 \lambda_{p}, r_{e}, r_{h}\right)-2 \lambda_{p}(\delta+1) D_{3}\left(2 \lambda_{p}, r_{e} r_{h}\right)\right\} \\
A_{e, p}=\phi_{e, p}^{2}(R) \int_{0}^{\infty} d r_{h} r_{h} \phi_{h, p}^{2}\left(r_{h}\right) S_{2}\left(2 \lambda_{p}, R, r_{h}\right), & \\
B_{e, p}=\int_{R}^{\infty} d r_{e} \int_{0}^{\infty} d r_{h} r_{e} r_{h} \phi_{e, p}^{2}\left(r_{e}\right) \phi_{h, p}^{2}\left(r_{p}\right)\{ & {\left[(1+\delta)^{2}+\lambda_{p}^{2}\left(r_{e}^{2}+r_{h}^{2}\right)\right] F_{3}\left(2 \lambda_{p}, r_{e}, r_{h}\right) } \\
& \left.-2 \lambda_{p}^{2} r_{e} r_{h} F_{4}\left(2 \lambda_{p}, r_{e}, r_{h}\right)-2 \lambda_{p}(\delta+1) D_{3}\left(2 \lambda_{p}, r_{e}, r_{h}\right)\right\}
\end{aligned}
$$

and, correspondingly, $A_{h, p}=\left.A_{e, p}\right|_{e \leftrightarrow h}, B_{h, p}=\left.B_{e, p}\right|_{e \leftrightarrow h}$. We also defined

$C_{p}=\int_{0}^{\infty} d r_{e} \int_{0}^{\infty} d r_{h} r_{e} r_{h} \phi_{e, p}^{2}\left(r_{e}\right) \phi_{h, p}^{2}\left(r_{h}\right)\left[\delta^{2} G_{3}\left(2 \lambda_{p}, r_{e}, r_{h}\right)-2 \lambda_{p} \delta F_{3}\left(2 \lambda_{p}, r_{e}, r_{h}\right)+\lambda_{p}^{2} D_{3}\left(2 \lambda_{p}, r_{e}, r_{h}\right)\right]$,

$N_{p}=\int_{0}^{\infty} d r_{e} \int_{0}^{\infty} d r_{h} r_{e} r_{h} \phi_{e, p}^{2}\left(r_{e}\right) \phi_{h, p}^{2}\left(r_{h}\right)\left\{\left[\delta^{2}+\lambda_{p}^{2}\left(r_{e}^{2}+r_{h}^{2}\right)\right] F_{3}\left(2 \lambda_{p}, r_{e}, r_{h}\right)-2 \lambda_{p}^{2} r_{e} r_{h} F_{4}\left(2 \lambda_{p}, r_{e}, r_{h}\right)-2 \lambda_{p} \delta D_{3}\left(2 \lambda_{p}, r_{e}, r_{h}\right)\right\}$, 
and

$$
\begin{aligned}
S_{2}\left(\lambda, x_{1}, x_{2}\right)=\frac{\partial}{\partial x_{1}} \frac{1}{x_{1}}\{ & {\left[\delta^{2}+\lambda^{2}\left(x_{1}^{2}+x_{2}^{2}\right)\right] F_{3}\left(\lambda, x_{1}, x_{2}\right) } \\
& -2 \delta \lambda D_{3}\left(2 \lambda, x_{1}, x_{2}\right) \\
& \left.-2 \lambda^{2} x_{1} x_{2} F_{4}\left(2 \lambda, x_{1}, x_{2}\right)\right\}
\end{aligned}
$$

where $F_{i}$ and $D_{i}$ are given by Eqs. (14) and (15), respectively.

\section{NUMERICAL RESULTS AND DISCUSSION}

We numerically evaluate the expressions derived in Sec. II and minimize $E_{s}$ and $E_{p}$ by variation of $\lambda_{s}$ and $\lambda_{p}$, respectively. For all our calculations we choose material parameters appropriate for $\mathrm{CdS}$, i.e., effective electron mass inside the quantum dot $m_{e, 1}=0.235 m_{0}$, effective hole mass $m_{h, 1}=1.35 m_{0}$, band gap $E_{g}=2.583 \mathrm{eV}$, exciton binding energy $E_{R}=27 \mathrm{meV}$, and exciton Bohr radius $a_{B}=30.1 \AA$. For simplicity, we neglect tunneling effects of the hole and choose $V_{0 h}=\infty$, i.e., the hole is confined inside the microcrystallites. To describe hole tunneling would require additional information about the occupied electron states outside the quantum dots, which is not available at the present time.

In Fig. 1 we plot the energies of the two quantumconfined states as function of quantum-dot radius for a confinement potential $V_{0 e}=40 E_{R}$ and $m_{e, 2}=m_{0}$. This situation is more or less appropriate for quantum dots in a liquid solution, where we assume that the electrons can move freely once they have tunneled out of the crystallites. The solid lines are the results obtained for infinite confinement potential, i.e., ideal quantum confinement, and the dashed lines are the results of our variational cal-

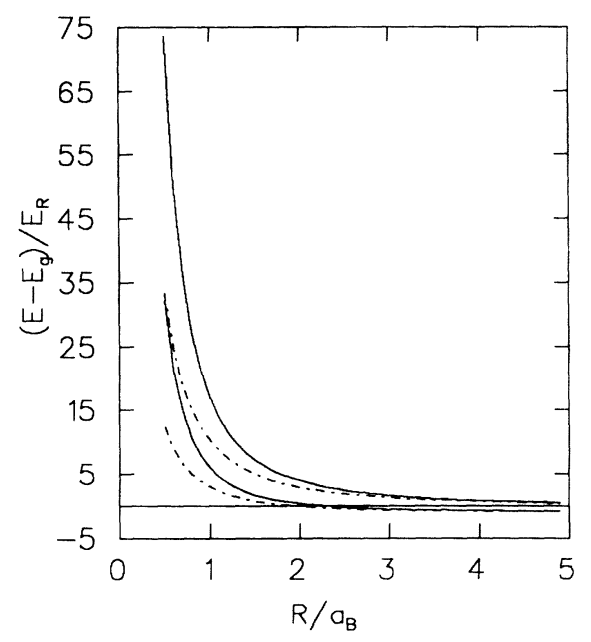

FIG. 1. Computed energies of the two lowest dipole-allowed quantum-confined states are plotted as functions of crystallite radius. The solid lines show the results for an infinite confinement potential and the dashed lines are for a confinement potential $V_{0}=40 E_{R}$. The other material parameters are $m_{e, 1}=0.235 m_{0}, m_{e, 2}=m_{0}, m_{h, 1}=1.35 m_{0}, E_{g}=2.583$ $\mathrm{eV}, E_{R}=27 \mathrm{meV}$, and $a_{B}=30.1 \AA$. culations. As expected, the lower potential barrier reduces the quantum-confined energy levels. The relative changes are larger for the excited state. This is due to the reduced confinement energy contribution for the case of a finite well height. This reduction is more pronounced for energetically higher states. For radii $R$ smaller than $\cong 0.4 a_{B}$ our calculations predict the disappearance of an excited state that is bound inside the quantum dot. In the limit $R \gg a_{B}$ our results approach the correct bulk semiconductor values of $-E_{R}$ for the ground state and $-E_{R} / 4$ for the excited state, respectively.

To demonstrate the dependence of the energy states on the strength of the confinement potential we plot in Fig. 2 the computed energy levels as function of $V_{0 e}$ assuming a crystallite radius $R=0.5 a_{B}$ and otherwise the same conditions as in Fig. 1. Figure 2 shows that both energy levels increase with increasing quantum confinement. The increase of the excited state is clearly more pronounced than that of the ground-state level. For a confinement potential which is less than $\cong 30 E_{R}$ the first excited state is no more bound inside the quantum dot for the chosen parameters.

In Fig. 3 we plot the dependence of the energy levels on the electron mass outside the semiconductor material for $R=0.5 a_{B}$ and $V_{0 e}=80 E_{R}$. We see that the energies decrease with increasing outside mass. This indicates that for quantum dots in glass, where the electrons outside are most likely in localized states simulated by a very heavy mass, the confined energy levels are lower than those for the same size quantum dots in a liquid solution. We verified the overall energy variation as function of mass ratio by solving the Schrödinger equation for a one-dimensional potential well with different masses inside and outside, using the same boundary conditions discussed above, i.e., $\phi_{i}\left(r_{i}\right)$ and $1 / m_{i} \partial \phi_{i}\left(r_{i}\right) / \partial r_{i}$ are continuous at $r_{i}=R$. We always find that the energy of the confined states decreases with increasing outside mass.

In Fig. 4 we compare our ground-state energies for

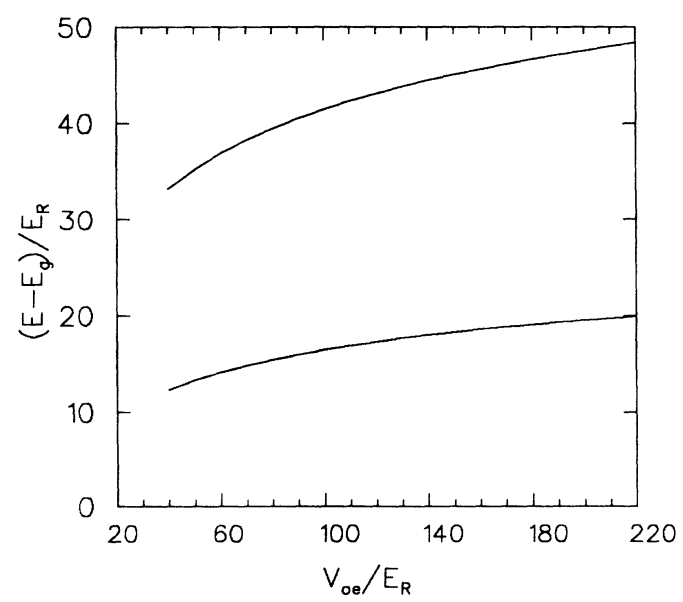

FIG. 2. Computed energies of the two lowest quantum confined states as functions of the confinement potential for a quantum dot of radius $R=0.5 a_{B}$. The other parameters are the same as in Fig. 1. 


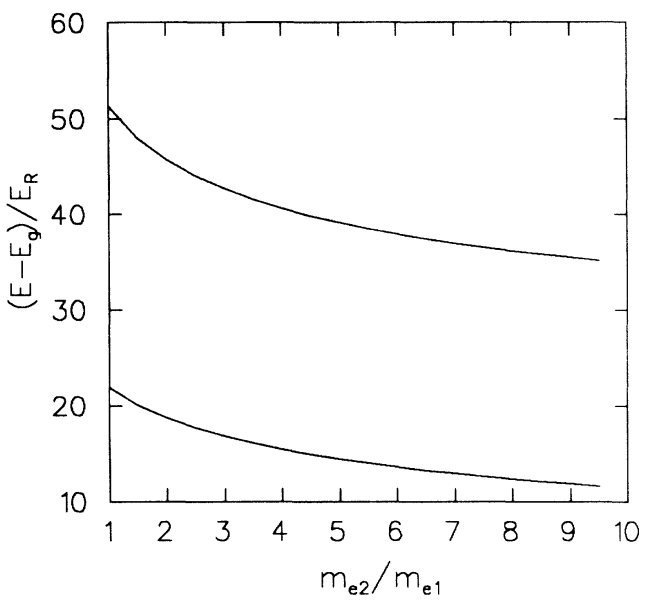

FIG. 3. Computed energies of the two lowest quantum confined states as functions of the electron mass outside the quantum dot for $R=05 . a_{B}, V_{0 e}=80 E_{R}$, and otherwise the same parameters as in Fig. 1 .

$V_{0 e}=40 E_{R}$ (dashed line) and $V_{0 e}=\infty$ (solid line) to the experimental results for CdS colloids obtained by Weller et al. ${ }^{4}$ The radius of the CdS crystallites was determined by electron microscopy ${ }^{11}$ (triangles) or by the fluorescence quenching method (squares). ${ }^{4}, 12$ Our results for a finite-potential barrier show good agreement with the experimental observations, whereas the infinite-potentialbarrier calculation clearly overestimates the confinement energy.

In conclusion, we have calculated the energy of the two lowest quantum-confined states of an electron-hole pair in semiconductor clusters including the tunneling effects of the electron. We found reasonably good agreement between our theoretical results and experimental data indicating the importance of the confinement conditions. Recent calculations ${ }^{15,16}$ have shown that the effective-mass approximation especially for the holes, may not be very good due to the mixing between the heavy- and light-hole states in small quantum dots. Clearly, for a truly quanti-

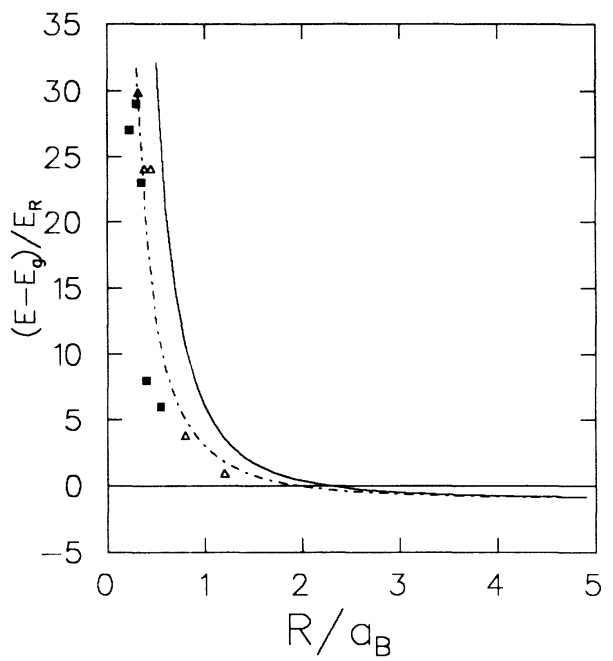

FIG. 4. Computed ground-state energy for a well depth $V_{0 e}=40 E_{R}$ (dashed line) and $V_{0 e}=\infty$ (solid line) in comparison with the experimental results of Weller et al. (Ref. 4). The parameters are the same as in Fig. 1.

tative analysis these modifications must be taken into account, in addition to the effects considered in the present paper.

\section{ACKNOWLEDGMENTS}

One of us (D.B.T.T) thanks Professor E. Zeitler for continuing interest and support and the Max-PlanckGesellschaft for partial support. The Arizona group would like to acknowledge helpful discussions with $\mathbf{M}$. Lindberg, financial support from National Science Foundation (Grants. No. ECS-89-09913, No. ECS-88-22305, and No. INT-87-13068 travel grant). North Atlantic Treaty Organization (travel Grant No. 87/0736), the Optical Circuitry Cooperative at the University of Arizona, and a grant for CPU time from the Pittsburgh Supercomputer Center (Pittsburgh, PA).
${ }^{*}$ Permanent address: National Center for Scientific Research of Vietnam, Mac Dinh Chi 1, Ho Chi Minh City, Vietnam.

${ }^{1}$ Al. L. Efros and A. L. Efros, Fiz. Tekh. Poloprovodn. 16, 1209 (1982) [Sov. Phys. - Semicond. 16, 772 (1982)].

${ }^{2}$ L. E. Brus, J. Chem. Phys. 80, 4403 (1984).

${ }^{3}$ A. I. Ekimov and A. A. Onuschchenko, Fiz. Tekh. Poloprovodn. 16, 1215 (1982) [Sov. Phys. - Semicond. 16, 775 (1982)].

${ }^{4}$ H. Weller, H. M. Schmidt, U. Koch, A. Fojtik, S. Baral, A. Henglein, W. Kunath, K. Weiss, and E. Dieman, Chem. Phys. Lett. 124, 557 (1986).

${ }^{5}$ Y. Kayanuma, Solid State Commun. 59, 405 (1986).

${ }^{6}$ H. M. Schmidt and H. Weller, Chem. Phys. Lett. 129, 615 (1986).

${ }^{7}$ L. E. Brus, IEEE J. Quantum Electron. QE-22, 1909 (1986); for a recent review and further references see also $M$. Bawendi, M. Steigerwald, and L. Brus, Annu. Rev. Phys. Chem. (to be published).

${ }^{8}$ L. Banyai, Y. Z. Hu, M. Lindberg, and S. W. Koch, Phys. Rev. A 38, 8142 (1988).

${ }^{9}$ S. Schmitt-Rink, D. A. Miller, and D. S. Chemla, Phys. Rev. B 35, 8113 (1987).

${ }^{10}$ Y. Z. Hu, S. W. Koch, M. Lindberg, N. Peyghambarian, E. L. Pollock, and F. F. Abraham, Phys. Rev. Lett. 64, 1805 (1990); Y. Z. Hu, M. Lindberg, and S. W. Koch, Phys. Rev. B 42, 1713 (1990).

${ }^{11}$ W. Kunath, F. Zemlin, and K. Weiss, Ultramicroscopy 16, 123 (1985).

12J. J. Ramsden and M. Graetzel, J. Chem. Soc. Faraday Trans. 
I 80, 919 (1984).

${ }^{13}$ Y. Wang, N. Herron, W. Mahler, and A. Suna, J. Opt. Soc. Am. B 6, 808 (1989).

${ }^{14}$ S. H. Park, R. A. Morgan, Y. Z. Hu, M. Lindberg, S. W.
Koch, and N. Peyghambarian, J. Opt. Soc. Am. B 7, 2097 (1990).

${ }^{15}$ Jian-Bai Xia, Phys. Rev. B 40, 8500 (1989).

${ }^{16}$ P. C. Sercel and K. J. Vahala, in Phys. Rev. B 42, 3690 (1990). 